Proceeding of the $1^{\text {st }}$ ICEENG conference, 24-26 March, 1998.

$B E-3 \quad 5 \angle 5$

MILITARY TECHNICAL COLLEGE
CAIRO-EGYPT

\title{
IMAGE COMPRESSION USING VECTOR QUANTIZATION IN THE WAVELET DOMAIN
}

\author{
A. S. Ragab*, Abdalla S. A. Mohamed**, M. S. Hamid ${ }^{* *}$
}

\begin{abstract}
Image compression becomes the main task for reducing the image bit rate with acceptable reconstructed image quality. Wavelet transform shows a good way of subdividing the image into different subbands with different resolutions which are quantized to reduce the inage bit rate. This paper introduces the application of Vector qauntization (VQ) to the image subbands in the wavelet ciomain. The image spectrum is decomposed into hierarchical subbands using the wavelet transform. Three methods of codebook generation are discussed namely, global codebook, local codebook, and variance based codebook with application to image subbands for different block sizes. The quantized coefficients and the baseband are Hufmann coded. Results showed that the local codebook based method gave better peak signal to noise ratio (PSNR) than the global codebook with nearly the same compression ratio (CR). But for variance based method, the visual quality of the reconstructed image is better than those of the two other methods for nearly the same PSNR, and CR as the edges are preserved.
\end{abstract}

\section{KEY WORDS}

Image compression - Wavelets - Vector Quantization.

\section{INTRODUCTION}

The Wavelet transform [1] becomes a good way of subdividing the image into different frequency subbands with different resolutions. Vector quantization has been demonstrated to be an efficient method for image compression. The image spectrum is decomposed into seven hierarchical subbands and the LBG vector quantization algorithm, proposed by Linde, Buzo, and Gray [2] is applied to the image subbands with three methods of codebook generation namely, global codebook, local codebook and variance

* Ph. D, Dpt. of Electronic Engineering, Military Technical College, Cairo, Egypt.

** Professor, Dpt. of Systems \& Biomedical Engineering, Cairo University, Cairo, Egypt.

*** Graduate student, Dpt. of Electronic Engineering, Military Technical College, Cairo, Egypt. 
based codebook. The quantized coefficients are coded by Hufmann technique. This paper is organized as follows. In Section II after a short introduction to wavelet transforms, the application of wavelet transform for subband filtering of the image is introduced. Section III describes three methods of generating the codebook for quantizing the image subbands using VQ and how these subbands are coded. Section IV presents the results and comparisons of encoding performance of the three methods using standard image. Concluding comments are presented in Section $\mathbf{V}$.

\section{DISCRETE WAVELET TRANSFORM AND IMAGE SUBBAND FILTERING}

The orthogonal wavelet transform [3] would be used to decompose the given data (signal or image) into both time (spatial) and spectrally local coefficients. For applying the wavelet transform to images, the image is first treated as rows and then as columns. The continuous wavelet transform of a function $\mathrm{f}(\mathrm{t})$ is defined as:

$$
\mathrm{WT}(f(t), a, b)=\left\langle f(t), \frac{1}{\sqrt{\mathrm{a}}} \psi\left(\frac{(t-b)}{a}\right)\right\rangle
$$

Where $\psi(t)$ is so-called the mother wavelet. The parameter $\mathrm{b}$ is a time shift and the parameter a is a scaling of the time variable $t$.

Dyadic Wavelets : The dyadic wavelets are wavelets whose transform parameters have become discrete $a=2^{-i}$ and $b=2^{-i} k, i, k \in Z$ ( $Z$ is the set of integer numbers). For the discrete wavelet transform, signals are projected into a finite number of subspaces. In this case they must be element of a proper subspace of $\mathrm{L}_{2}(\mathbb{R}): f(t) \in \mathrm{V}_{0} \subset \mathrm{L}_{2}(\mathbb{R})$.

Where

$$
\mathrm{V}_{\mathrm{o}}=\mathrm{V}_{\mathrm{j}} \oplus \mathrm{W}_{\mathrm{j}} \oplus \mathrm{W}_{\mathrm{j}+1} \oplus \mathrm{W}_{\mathrm{j}+2} \oplus \ldots \oplus \mathrm{W}_{\mathrm{o}}
$$

and

$$
\mathrm{V}_{\mathrm{i}+1}=\mathrm{V}_{\mathrm{i}} \oplus \mathrm{W}_{\mathrm{i}}
$$

The index $\mathrm{i}$ is arbitrary and depends on the depth of decomposition.

Then, there exists a so-called scaling function $\varphi_{i, k}(t)$ which together with its shifts and translates, $\varphi_{i, k}(t)=2^{i / 2} \varphi\left(2^{i} t-k\right)$ constitutes an orthonormal basis systems of all spaces $V_{i}$. The decomposition of a signal using wavelet transform is given by,

$$
\begin{gathered}
f(t)=\sum_{i} \sum_{k} c_{i, k} \cdot \psi_{i, k}(t) \quad, \mathrm{i}, \mathrm{k} \in \mathbf{Z} \\
\psi_{i, k}(t)=2^{i / 2} \psi\left(2^{i} t-k\right)
\end{gathered}
$$

Where $c_{i, k}$ are calculated using equation (1). 
$\psi\left(2^{i} t-k\right)$ are scaled and shifted versions of $\psi(t)$ and $2^{1 / 2}$ ensures that $\left\|\psi_{i, m}(t)\right\|$ is always unity. The computation of the basic vectors $\varphi_{i, m}(t)$ and $\psi_{i, m}(t)$ using a quadrature mirror filter is given by the following recursive relations;

$$
\begin{gathered}
\varphi_{i, m}(t)=\sum_{n} h_{o}(n-2 m) \cdot \varphi_{i+1, n}(t), m, n \in \mathbf{Z} \\
\psi_{i, m}(t)=\sum_{n} h_{1}(n-2 m) \cdot \varphi_{i+1, n}(t)
\end{gathered}
$$

Where $h_{o}$ and $h_{l}$ are the coefficients of a quadrature mirror filter.

To apply the discrete wavelet transform to the image we used the hierarchical wavelet lecomposition suggested by Mallat [4]. The filter coefficients of Daub.6 [3] are used. The filter oefficients of $h_{o}$ and $h_{l}$ are applied to the image in both the horizontal and vertical directions and heir outputs are down sampled by 2 , generating the three selective orientations high subbands $. \mathrm{H}, \mathrm{HL}$, and $\mathrm{HH}$ and the low passband LL. Two levels of decomposition of the image for which ${ }_{1}=0$ and 1 are made yielding the seven hierarchical subbands $L_{1}, L_{1} H_{1}, H_{1}, H_{1}, L_{1}, H_{0}$, and $\mathrm{IH}_{0}$. The lowest frequency subband $\mathrm{LL}_{1}$ is called the baseband because it contains the most nportant perceptual information. This is shown in Fig. 1.

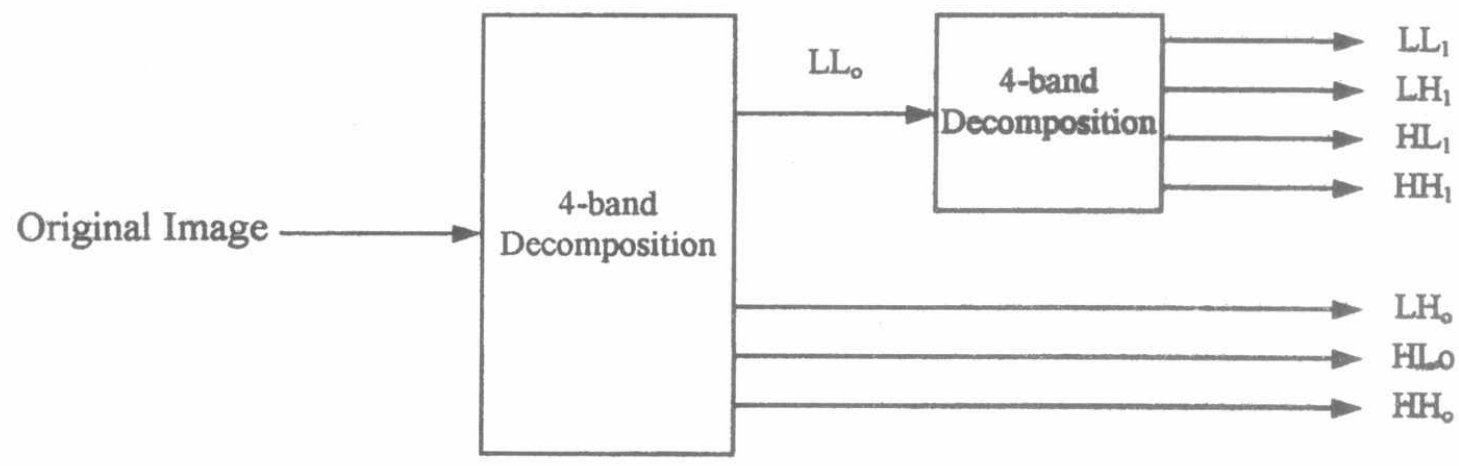

Fig. 1. Subband decomposition of the image.

\section{VECTOR QUANTIZATION AND CODING OF THE IMAGE SUBBANDS}

LBG vector quantization of image subbands showed a superior way to reduce the image bit rate with acceptable reconstructed image quality [5] compared with VQ applied to the image in the spatial domain. So, we splitted the image spectrum into seven hierarchical 
Proceeding of the $1^{\text {st }}$ ICEENG conference, $24-26$ March, 1998.

subbands using wavelet transform. The image subbands are divided into blocks of dimension $\mathrm{M} \times \mathrm{M}$ then LBG algorithm is applied. Due to the fact that the generated codebook to quantize the subbands has a great effect on the image quality, three methods for generating the codebook are discussed namely, local codebook, global codebook, and variance based codebook. In the local codebook method each subband is quantized with its own codebook generated from its vectors. While in the global codebook design, a codebook is generated for each resolution level containing the vectors selected from the three detailed subbands LH, HL, and $\mathrm{HH}$. The initial codebook of the local and global codebook methods are generated randomly with uniform distribution. The variance based codebook method [6] is generated by constructing a codebook for each subband. The vector dimension for this method must be as large as possible to ensure its randomness, e.g. a vector dimension of $32 \times 32$. The codebook vectors selected from the subband have the maximum variance of the whole subband vectors. Finally, the quantized subbands and the baseband are Hufmann coded.

\section{RESULTS}

The proposed procedure (VQ in the wavelet domain) is applied for standard image Lenna $512 \times 512(8 \mathrm{bpp})$ using the three methods of codebook generation.

For the global codebook method, as the vector dimension increases, the PSNR increases and the CR decreases except for block size $8 \times 8$ in which the PSNR is dropped by 2 $\mathrm{dB}$ and the $\mathrm{CR}$ is slightly increased. These results are shown in Figs. 2 and 3. The application of the local codebook method shows the increase of the PSNR with increasing the vector dimension and corresponding decrease of CR as shown in Figs. 4 and 5.

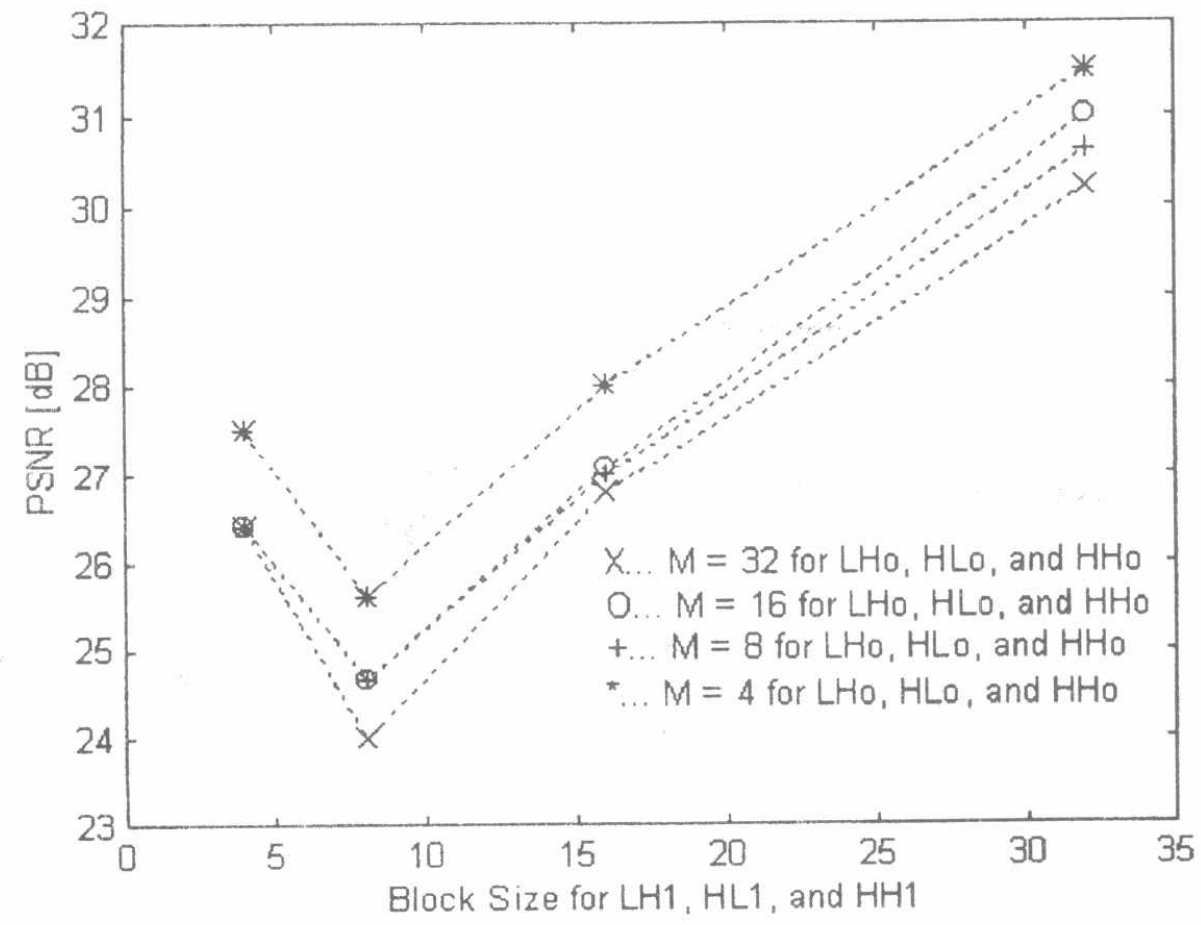

Fig. 2. PSNR against the block size for the global codebook method 
Although the values of PSNR and CR are nearly the same, the visual quality of the reconstructed image of the local codebook method is superior to that of the global codebook smoothing while the proposed method Because global codebook method results in edge shows nearly the same PSNR and CR aseserves edges. The variance based codebook best visual quality where the edges are preserved.

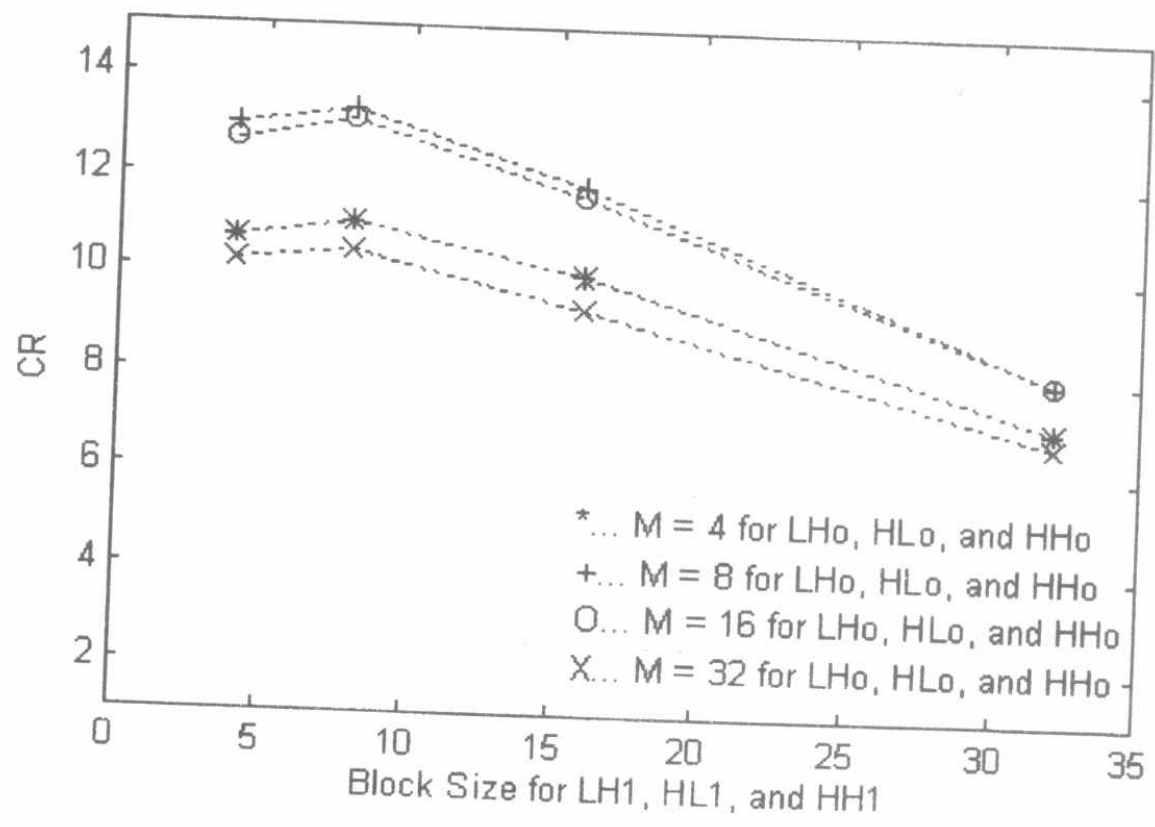

Fig. 3. CR against the block size for the global codebook method

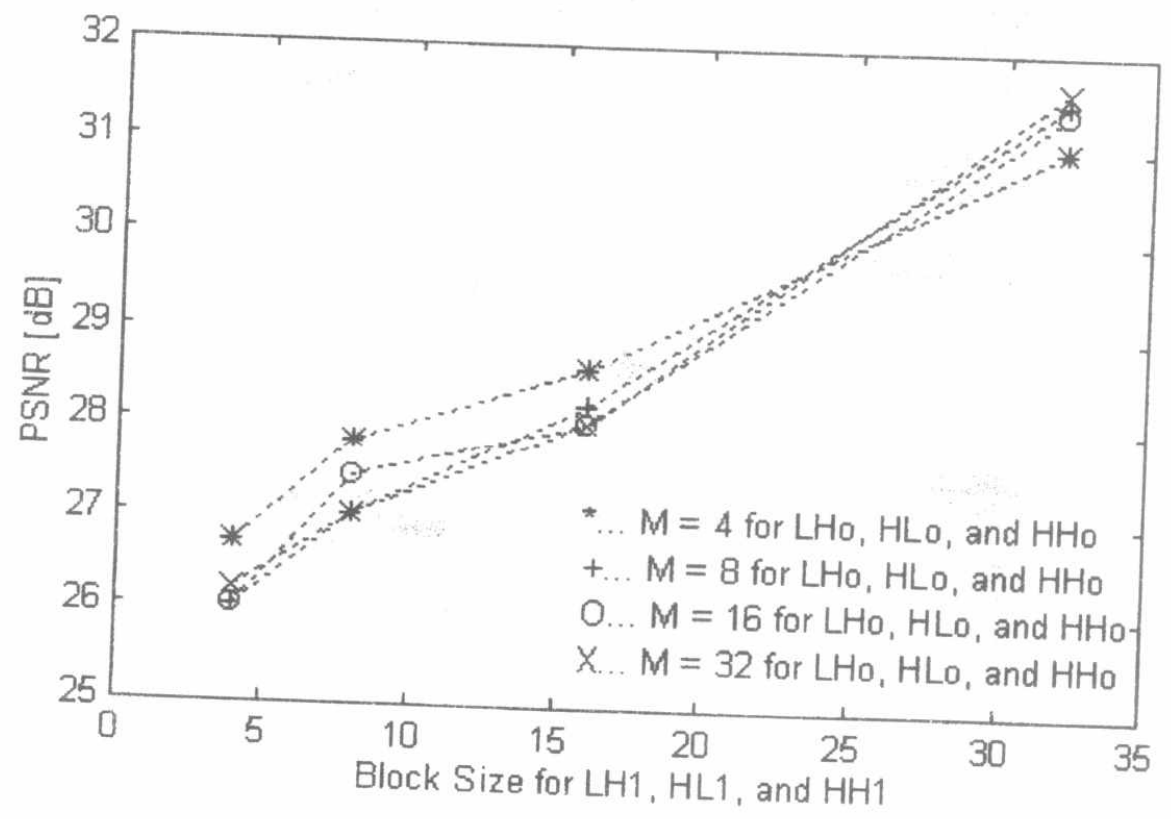

Fig. 4. PSNR against the block size for the local codebook method 
Proceeding of the $\mathbf{1}^{\text {st }}$ ICEENG conference, 24-26 March, 1998.

The reconstructed image after coding the image Lenna using the variance based method is shown in Figs. 11 and 12 in which we can recognize that, edges and detailed areas are better than those of the two other methods as shown in Figs. 11, 13 and 14

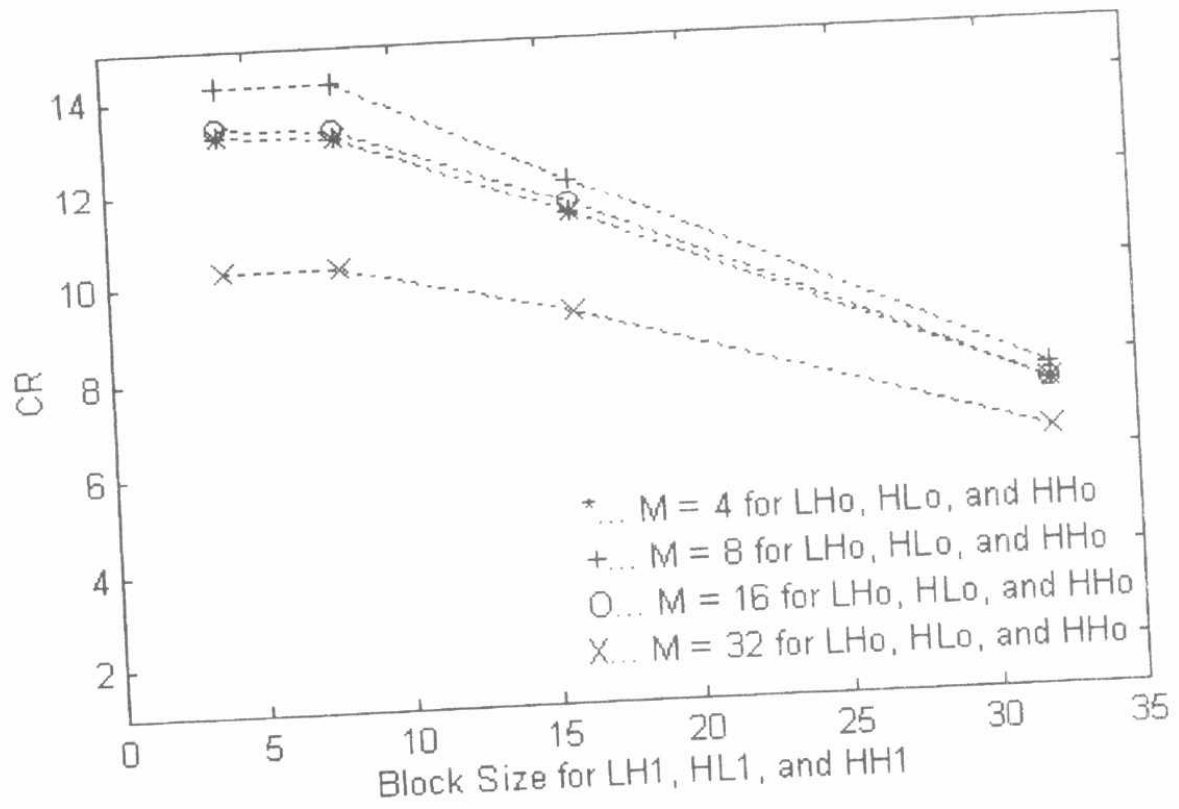

Fig. 5. CR against the block size for the local codebook method

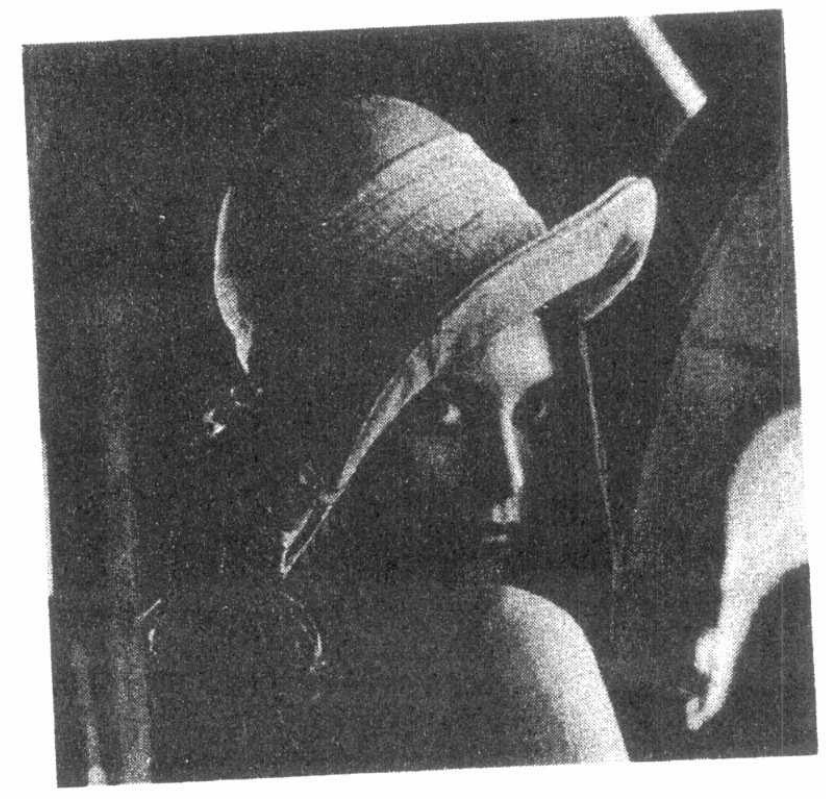

Fig. 6. Original image Lenna $512 \times 5128$ bpp. 


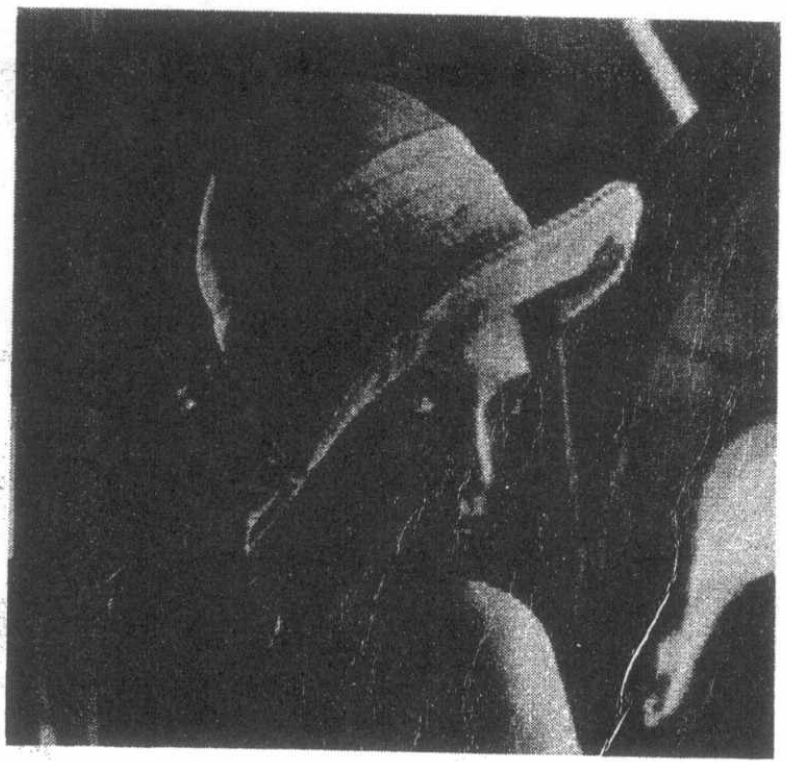

Fig. 7. Reconstructed image after applying the LBG algorithm with the global codebook.

$$
\mathrm{M}=8 \text { for } \mathrm{m}=0 \text { and } \mathrm{M}=4 \text { for } \mathrm{m}=1 \text {. }
$$$$
\text { PSNR }=26.5 \text { and } C R=13: 1 \text {. }
$$

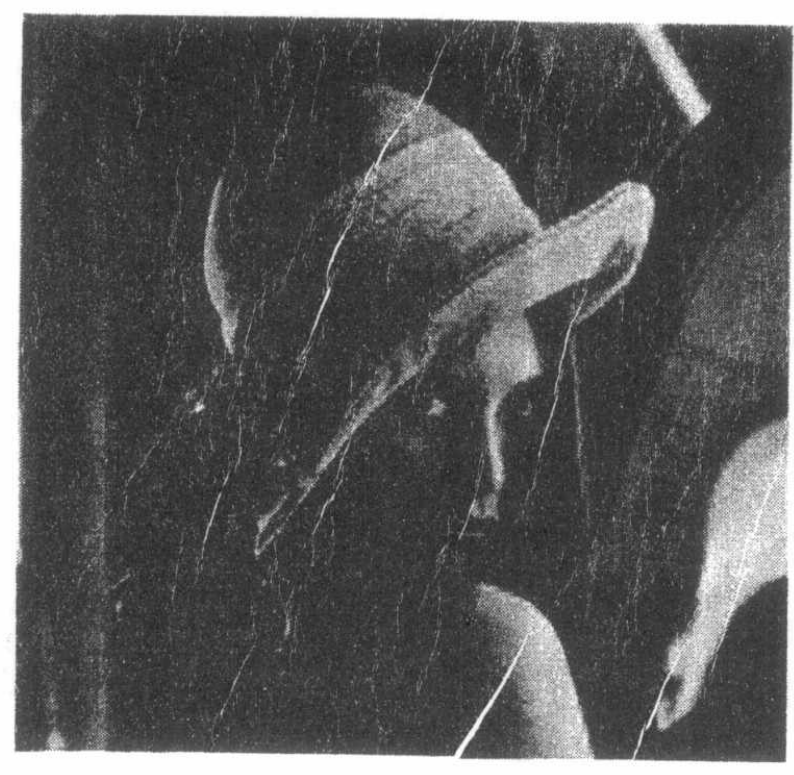

Fig. 9. Reconstructed irnage after applying the LBG algorithm with the local codebook. $M=8$ for $m=0$ and $M=4$ for $m=1$. $\mathrm{PSNR}=26 \mathrm{~dB}$ and $\mathrm{CR}=14: 1$.

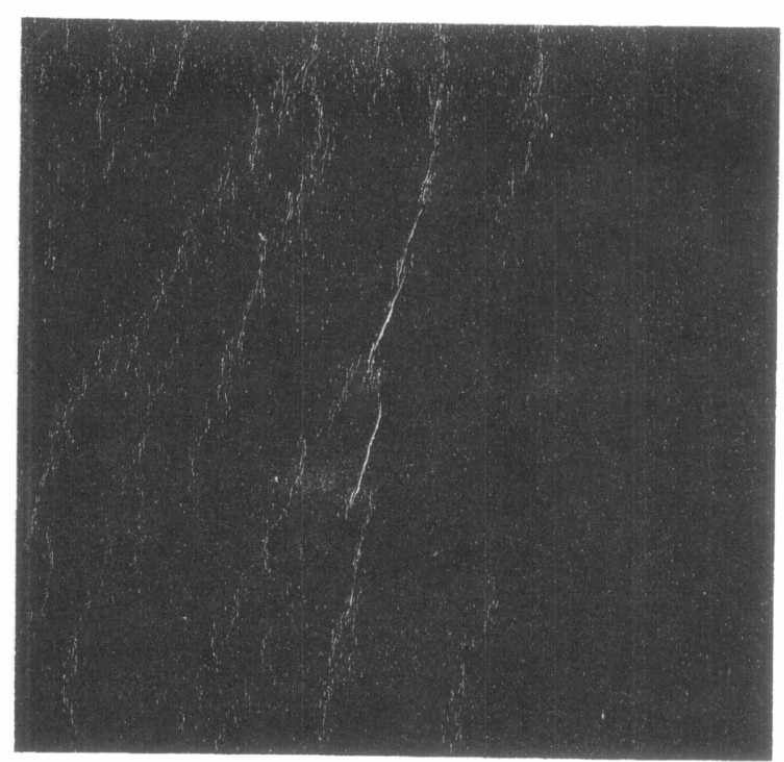

Fig. 8. Error image

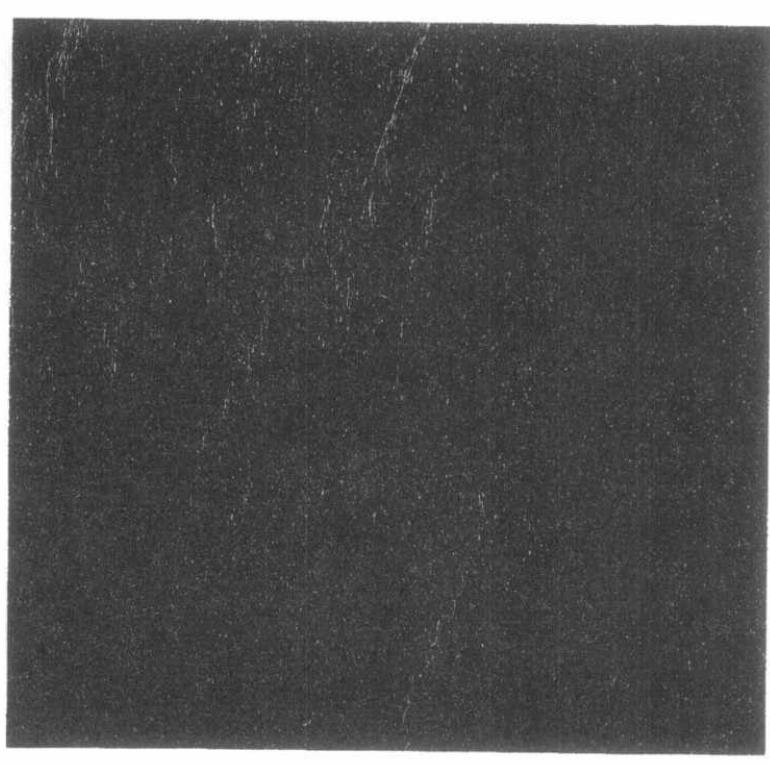

Fig.10. Error image. 


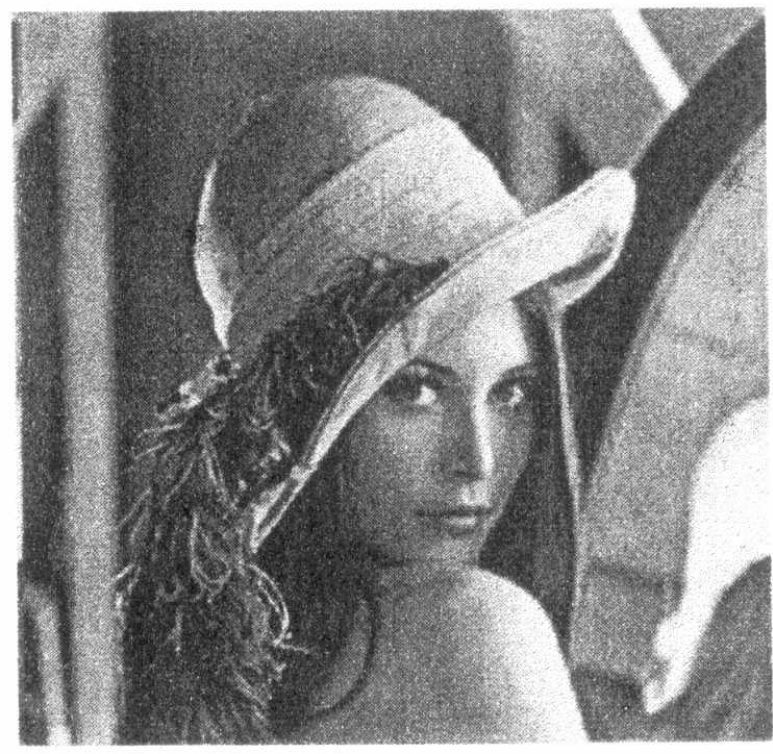

Fig. 11. Reconstructed image after applying the I. $B G$ algorithm with the variance based codebook $M=32$ for $m=0$ and $m=1$ PSNR $=31.3$ and $C R=6.4: 1$
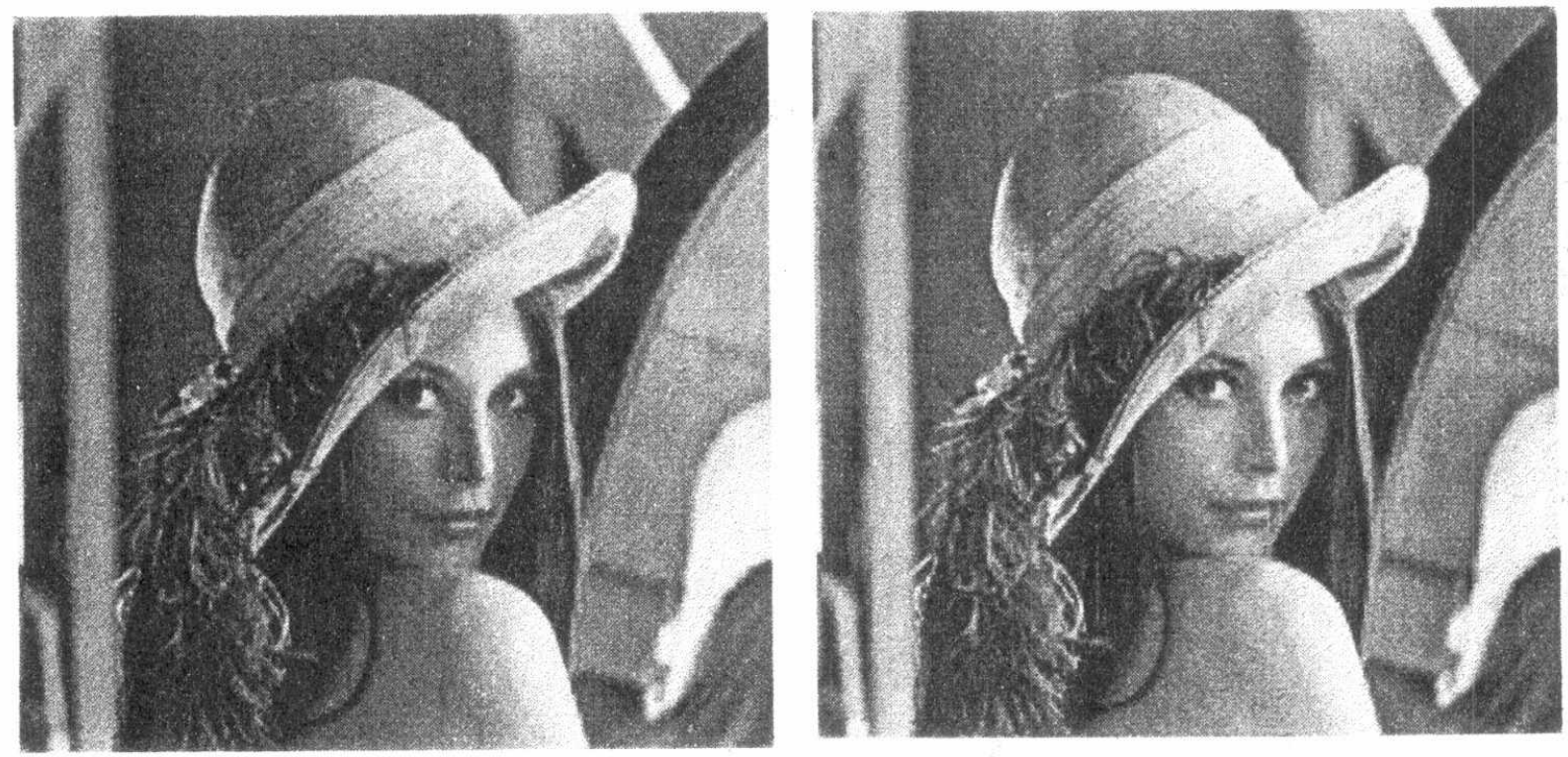

Fig. 13. Reconstructed image after applying the I.BG algorithm with the global codebook $M=32$ for $m=0$ and $m=1$.
$P S N R=30.2 d B, C R=6.7: 1$.

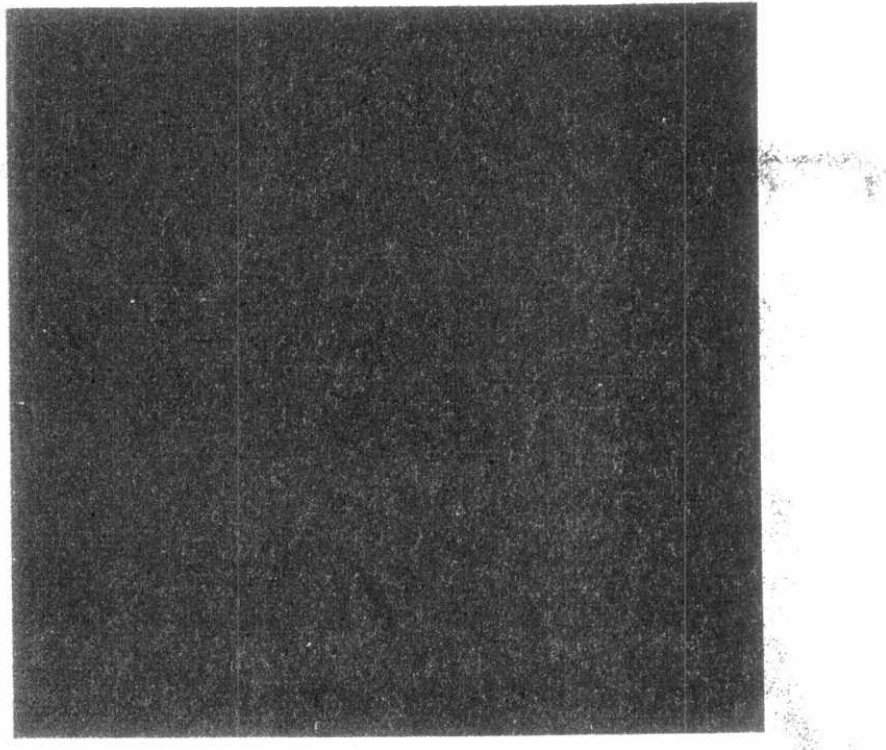

Fig. 12. Frror image 


\section{CONCLUSION}

To quantize images in the wavelet domain, it is better to construct a separate codebook for each subband to get a high perceptual quality. To preserve edges and high activity regions when using the LBG method to quantize the wavelet coefficients, variance based method should be used with the disadvantage of getting low CR which results from using a large vector dimension.

\section{REFERENCES}

[1] Marc Antonini, Michel Barlaud, Pierre Mathieu and Ingrid Daubechies, "Image Coding Using Wavelet Transform," IEEE Trans. on Image Processing, vol. 1, no. 2, (1992).

[2] Pamela C. C., Robert M. G., and Martin V., "Vector Quantization of Image Subbands : A Survey," IEEE Trans. on Image Processing, vol. 5, no. 2, February (1996).

[3] N. J. Fliege, 'Multirate Digital Signal Processing,' Hamburg University of Technology Germany, (1994).

[4] S. Mallat, "A Theory for Multiresolution Signal Decomposition: The Wavelet Representation," IEEE Trans. Pattern Anal., vol. 11, July (1989).

[5] Peter H., Dick E. Boekee, Jan Biemond, and John W. Woods, "Subband coding of images using vector quantization," IEEE Trans. Comm., vol. 36, no. 6, June (1988).

[6] Ruey-Feng Chang and Yu-Len Huang, "Finite-State Vector Quantization by Exploiting Interband and Intraband Correlations for Subband Image Coding," IEEE Trans. on Image Processing, vol. 5, no. 2, February (1996). 
